\title{
OPTIMAL DYNAMIC GREEN TIME FOR DISTRIBUTED
}

SIGNAL CONTROL

Kai Yuan, Victor L. Knoop, Serge P. Hoogendoorn

Department of Transport and Planning (TP), Delft University of Technology 


\section{Introduction}

- A variety of traffic signal control strategies for urban intersection exit;

$\square$ Isolated strategy

$\square$ Coordinated strategy 


\section{Distributed control \\ - Backpressure}

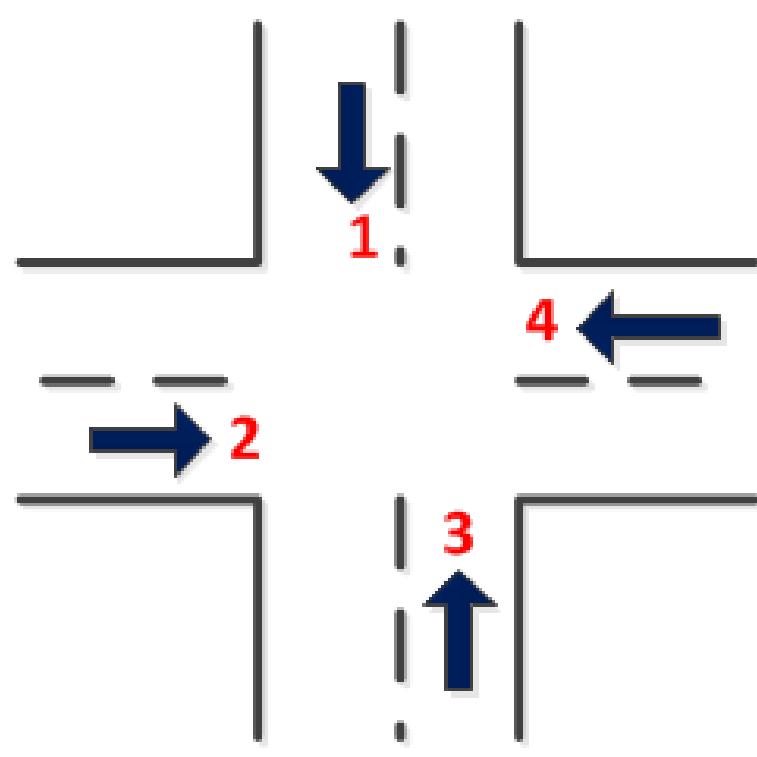

Every slot time, the intersection controller determines which phase to be activated, accordina to the local traffic situation 


\section{Distributed control \\ - Backpressure (s)}

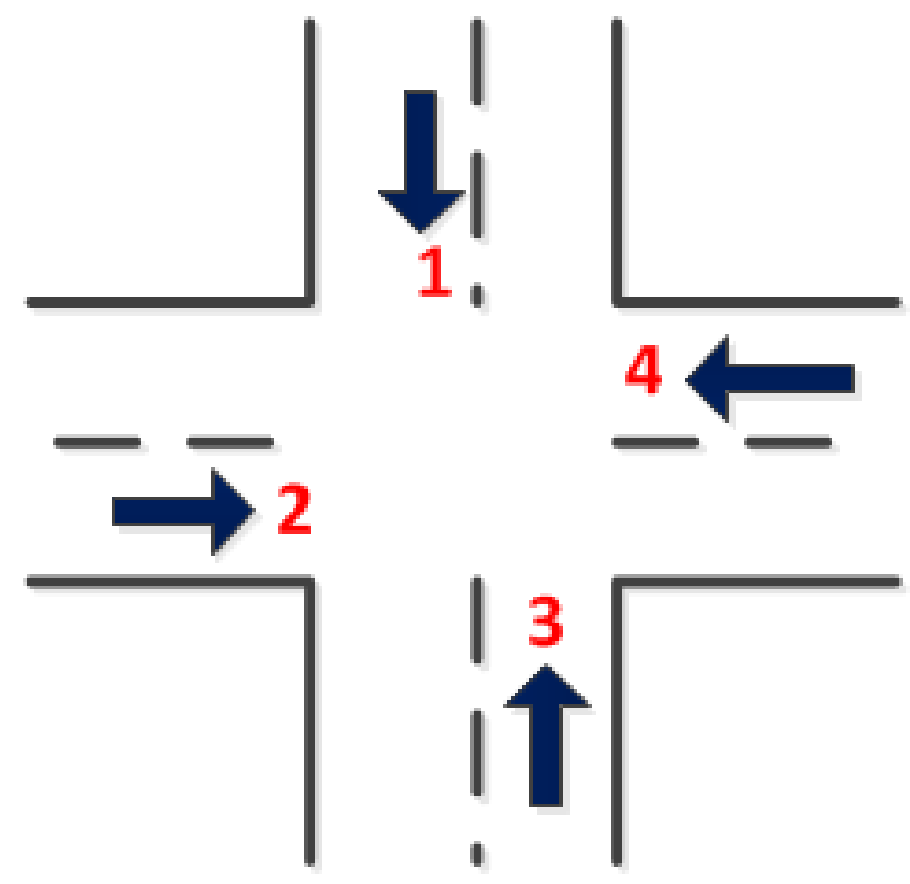

$$
\begin{gathered}
S(t) \square \quad W_{a b}(t) \xi_{i} \\
W_{a b}(t) \square Q_{a}(t)-Q_{b}(t)
\end{gathered}
$$




\section{Distributed control \\ - Backpressure}

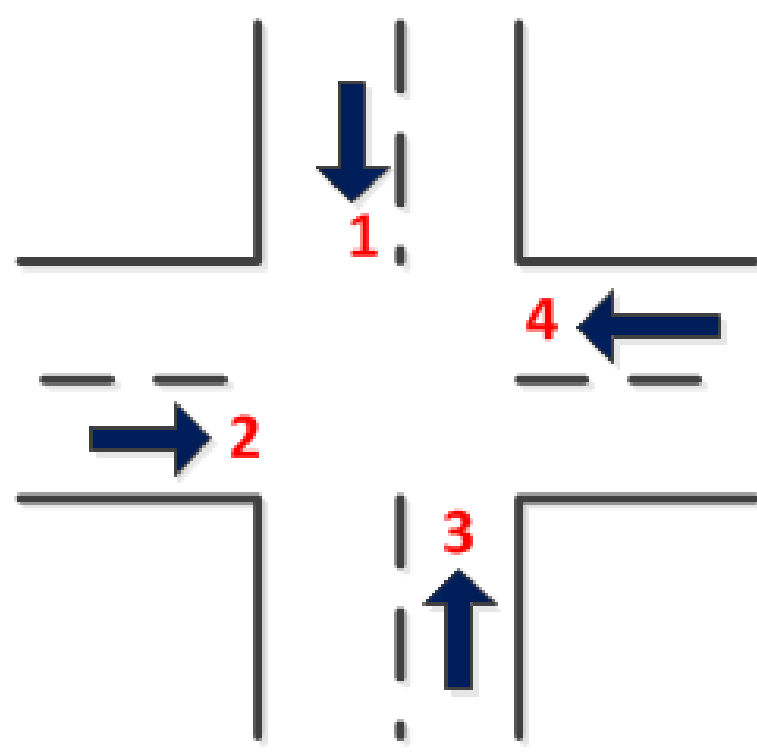

Every slot time, the phase with the highest the backpressure will be activated, e.g. given the right of the way. 


\section{Distributed control \\ - Backpressure}

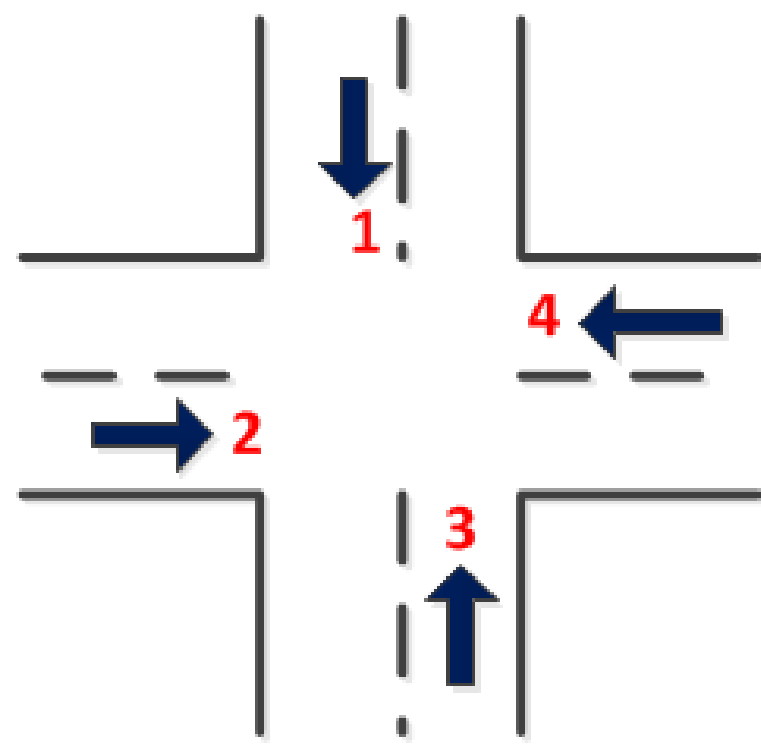

Slot time is the control step, and the green time length equals to (several) slot time length(es).

Wongpiromsarn, T., T. Uthaicharoenpong, W. Yu, E. Frazzoli, and W. Danwei. Distributed traffic signal control for maximum network throughput. In ITSC, $201215^{\text {TH }}$ IEEE Conference. 2012 
Problem:

1. "All red time" is not taken into consideration;

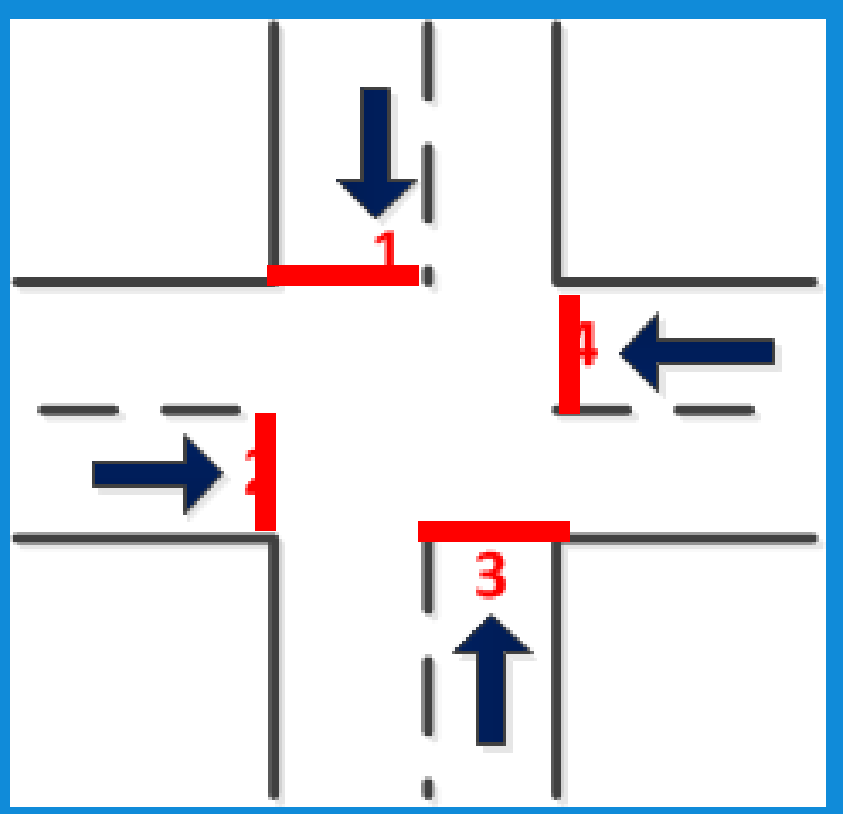




\section{Problem:}

2. Low robustness: possible large effect of a failing detector 
Therefore,

an optimal dynamic slot time approach is presented. 


\section{KEY CONCEPTS:}

$\left\{\begin{array}{l}\text { periodic } \\ \text { aperiodic }\end{array}\right\}$ control

$$
\begin{aligned}
& \left\{\begin{array}{l}
\text { static } \\
\text { dynamic }
\end{array}\right\} \text { slot time } \\
& \qquad\left\{\begin{array}{l}
\text { global } \\
\text { local }
\end{array}\right\} \text { slot time }
\end{aligned}
$$




\section{KEY CONCEPTS:}

$\left\{\begin{array}{l}\text { periodic } \\ \text { aperiodic }\end{array}\right\}$ control
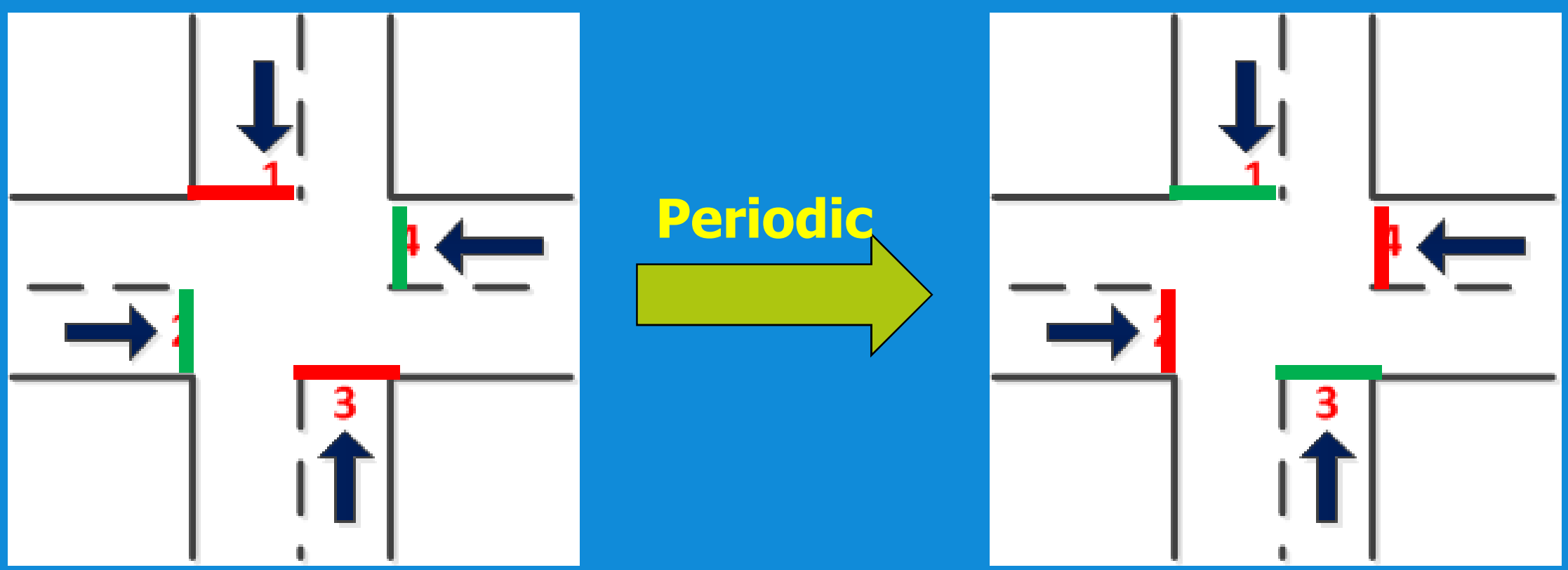


\section{KEY CONCEPTS:}

$\left\{\begin{array}{l}\text { periodic } \\ \text { aperiodic }\end{array}\right\}$ control
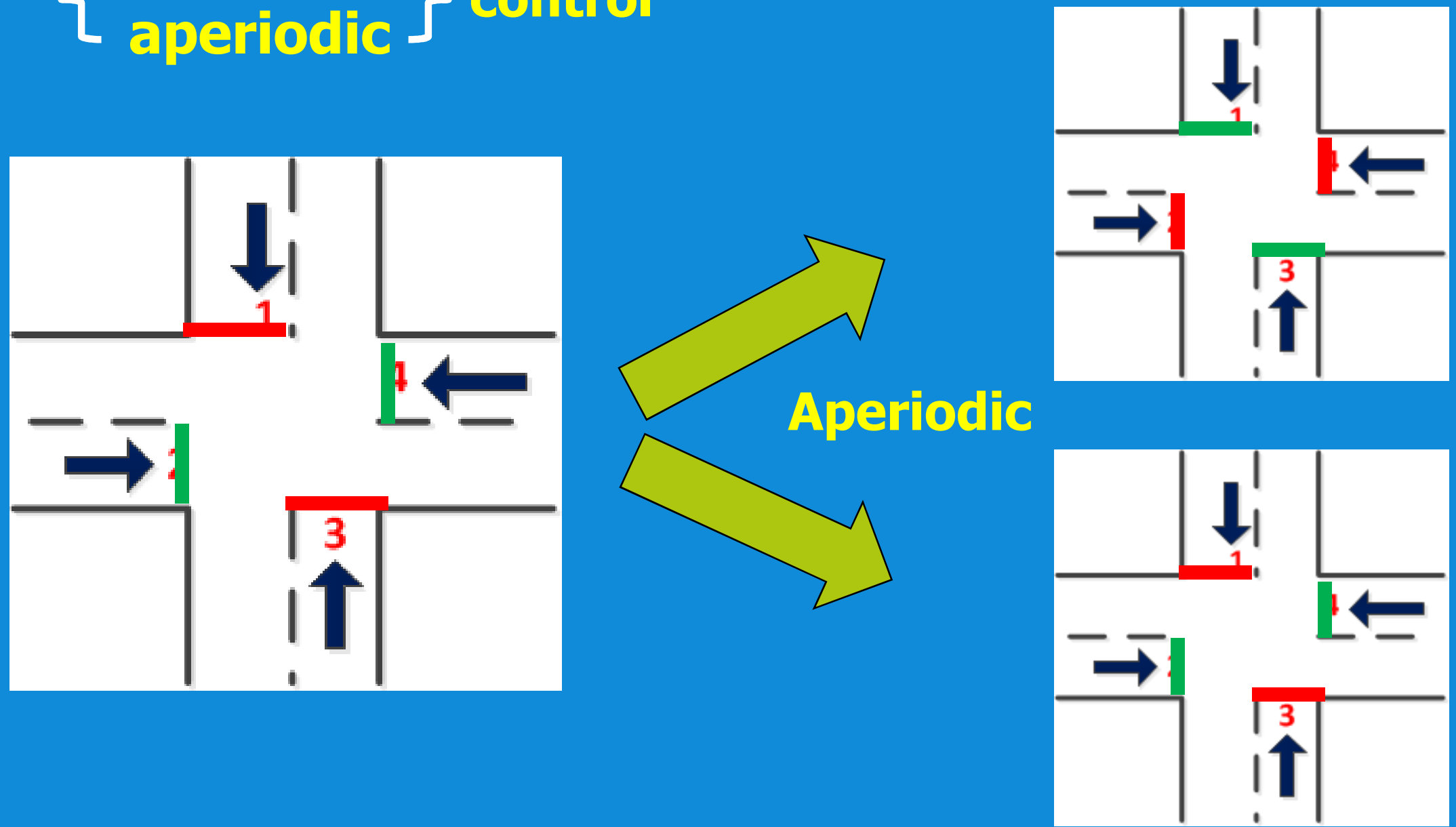


\section{KEY CONCEPTS:}

$\left\{\begin{array}{l}\text { periodic } \\ \text { aperiodic }\end{array}\right\}$ control

$$
\begin{aligned}
& \left\{\begin{array}{l}
\text { static } \\
\text { dynamic }
\end{array}\right\} \text { slot time } \\
& \qquad\left\{\begin{array}{l}
\text { global } \\
\text { local }
\end{array}\right\} \text { slot time }
\end{aligned}
$$




\section{KEY CONCEPTS:}

$\left\{\begin{array}{l}\text { periodic } \\ \text { aperiodic }\end{array}\right\}$ control

$$
\begin{aligned}
& \left\{\begin{array}{l}
\text { static } \\
\text { dynamic }
\end{array}\right\} \text { slot time } \\
& \qquad\left\{\begin{array}{l}
\text { global } \\
\text { local }
\end{array}\right\} \text { slot time }
\end{aligned}
$$

Critical junction: highest back-pressure or back-pressure difference 
Optimal green time approach 


\section{Dynamic slot time}
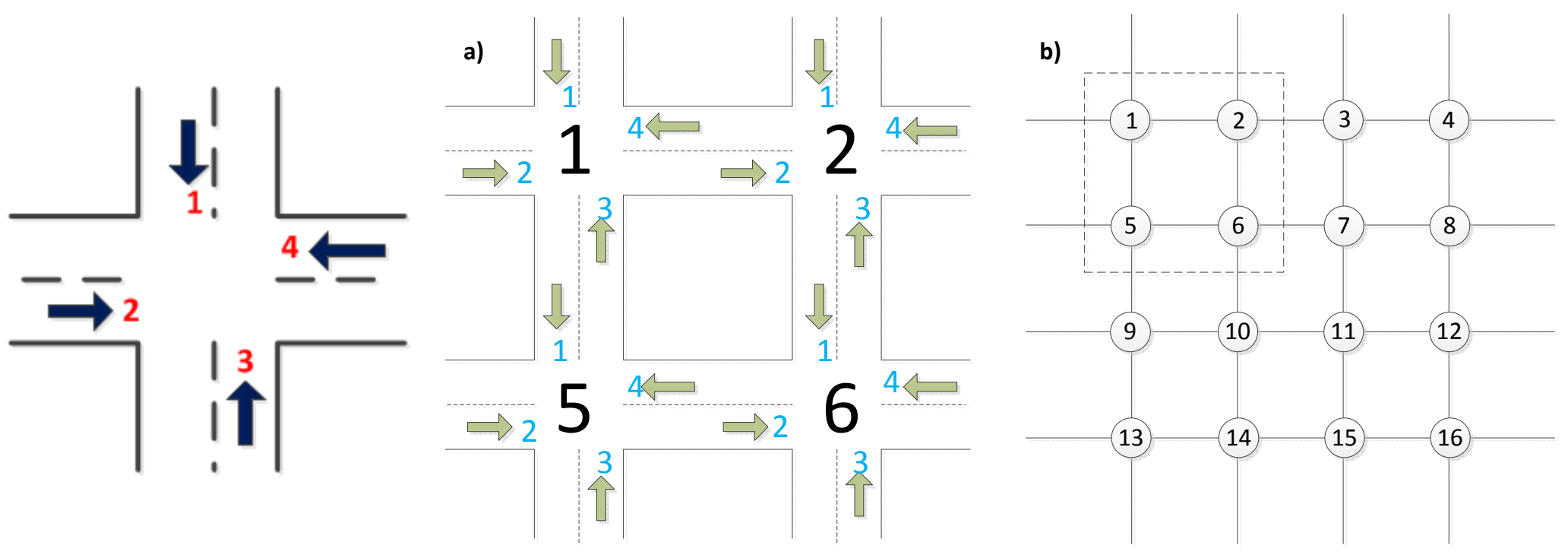


\section{Dynamic slot time}

$$
\begin{aligned}
& T_{\text {slot }}(t)=\tau+\max \left(0, \min \left(50, \tau_{A}(t)\right)\right) \\
& \begin{array}{l}
\text { Minimal green time } \\
\text { for each phase }
\end{array}
\end{aligned}
$$




$$
T_{\text {slot }}(t)=\tau+\max \left(0, \min \left(50, \tau_{A}(t)\right)\right)
$$

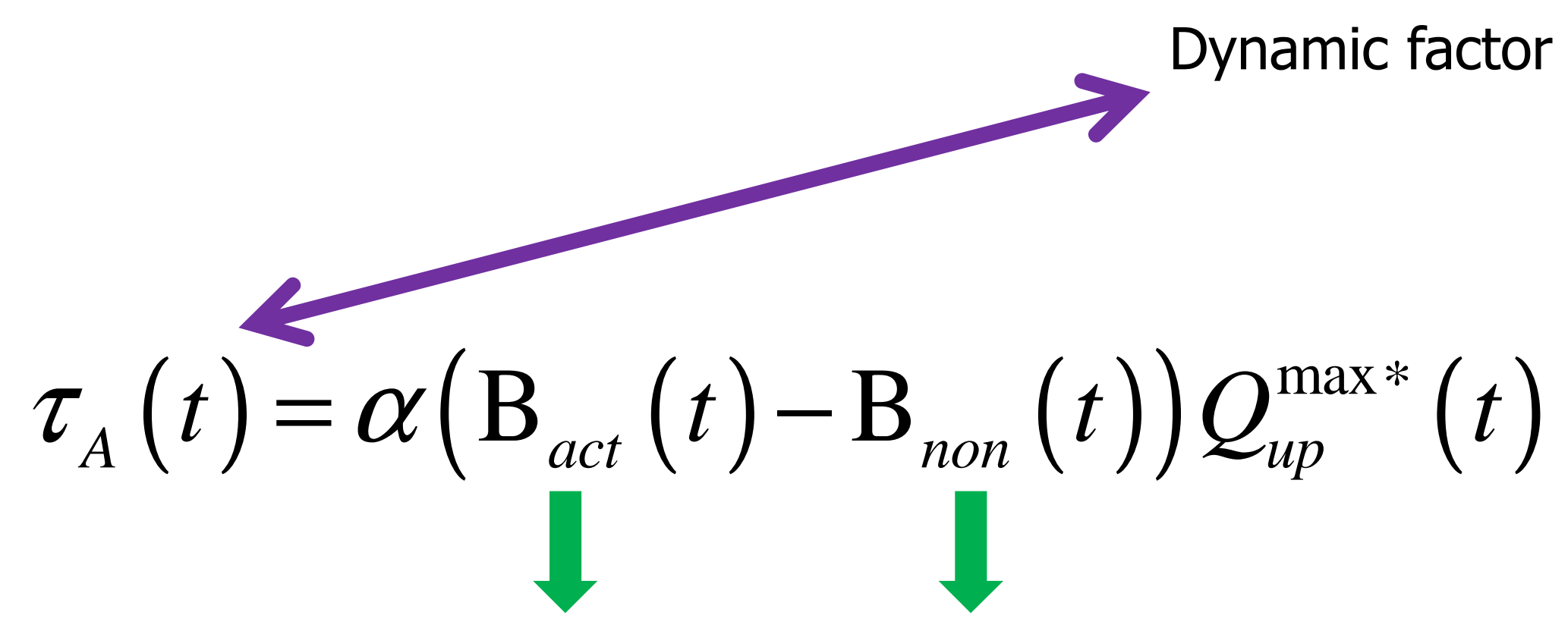

Backpressure of the Backpressure of the to be active phase next non-active phase 
Simulations 


\section{Network}
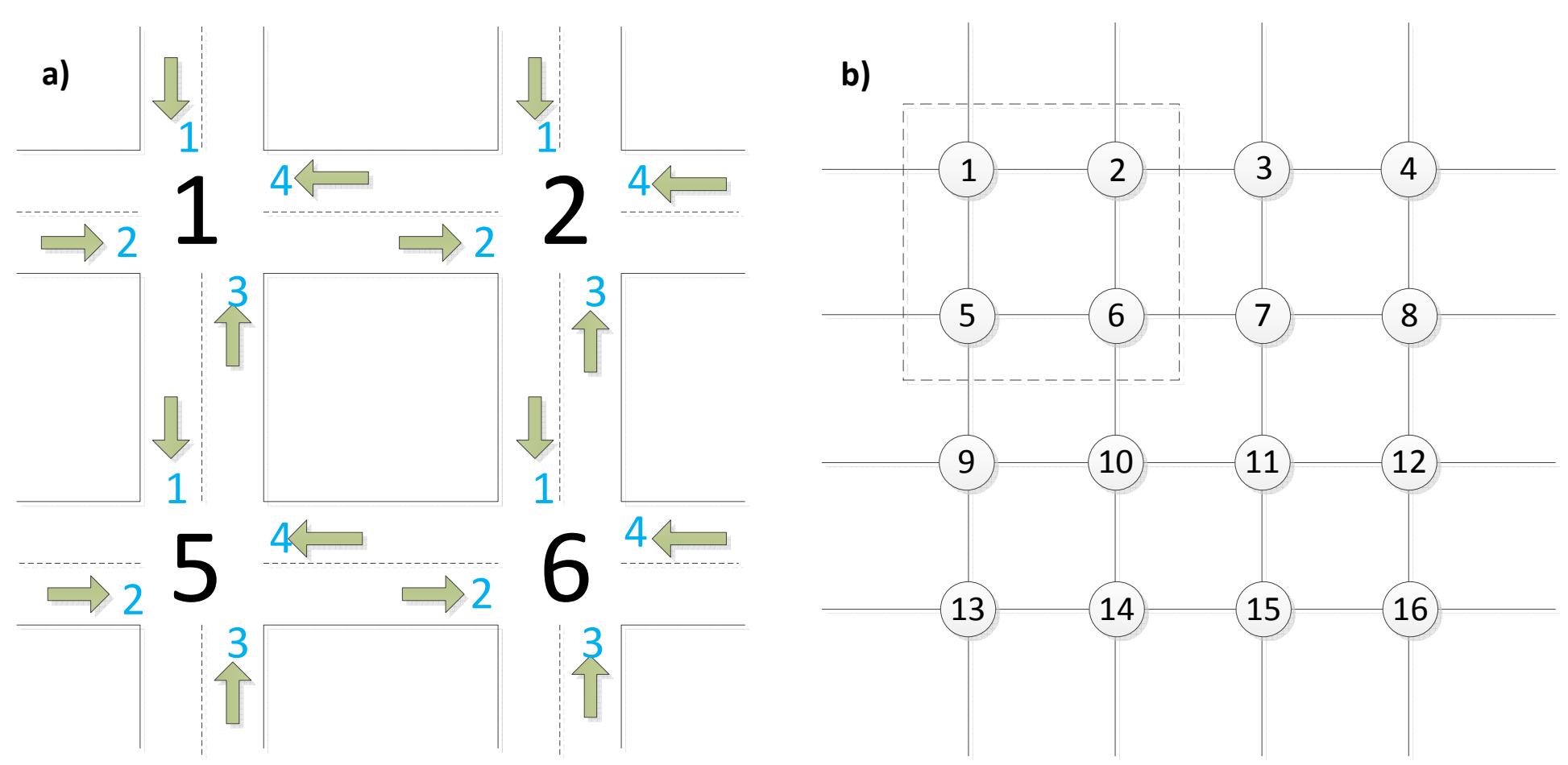


\section{Demand}

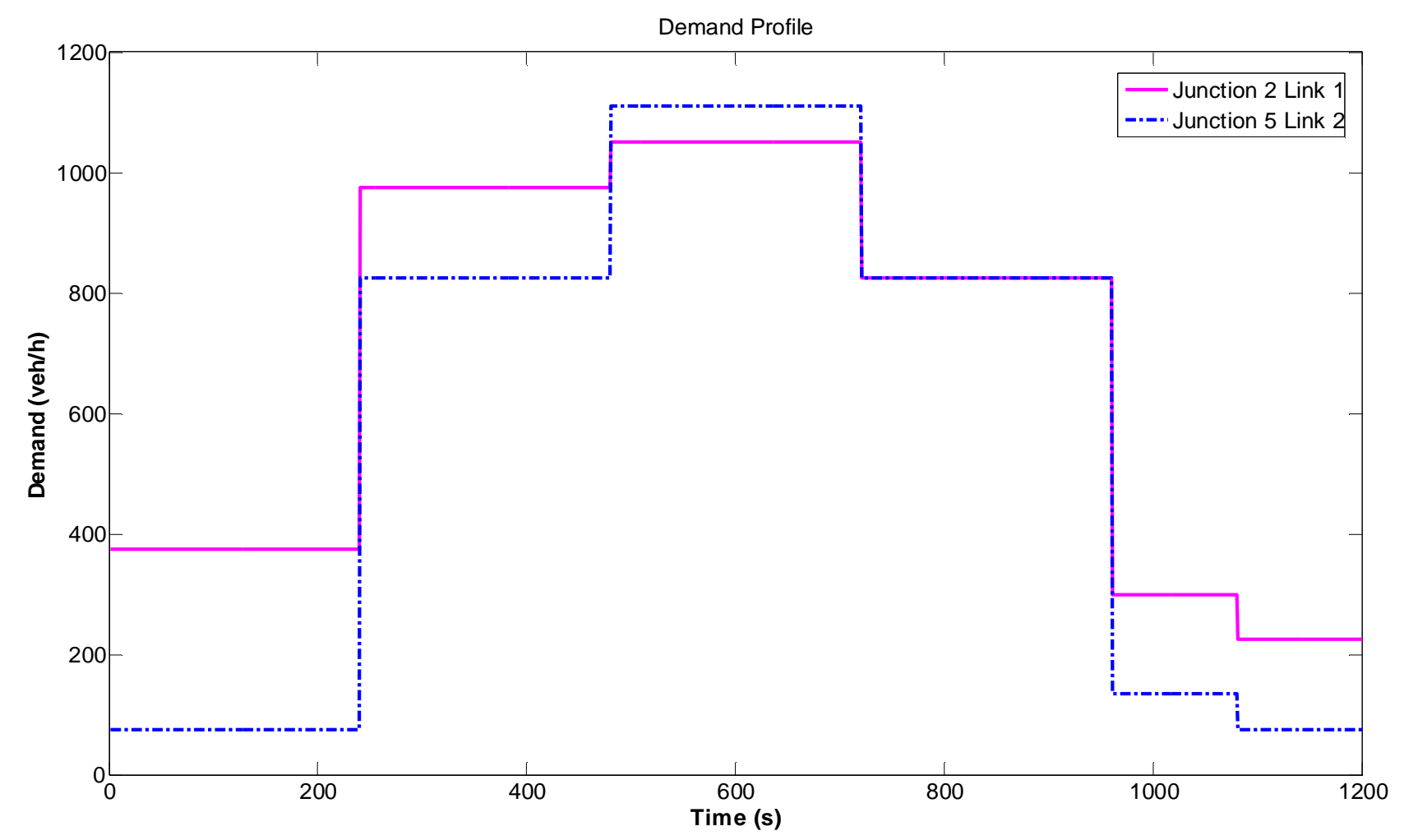




\begin{tabular}{|c|c|c|}
\hline Simulation scenarios & $\pi S$ & Max queue length \\
\hline Aperiodic & $2.9 \times 105$ & 21.07 \\
\hline periodic & $1.2 \times 105$ & 21.02 \\
\hline
\end{tabular}

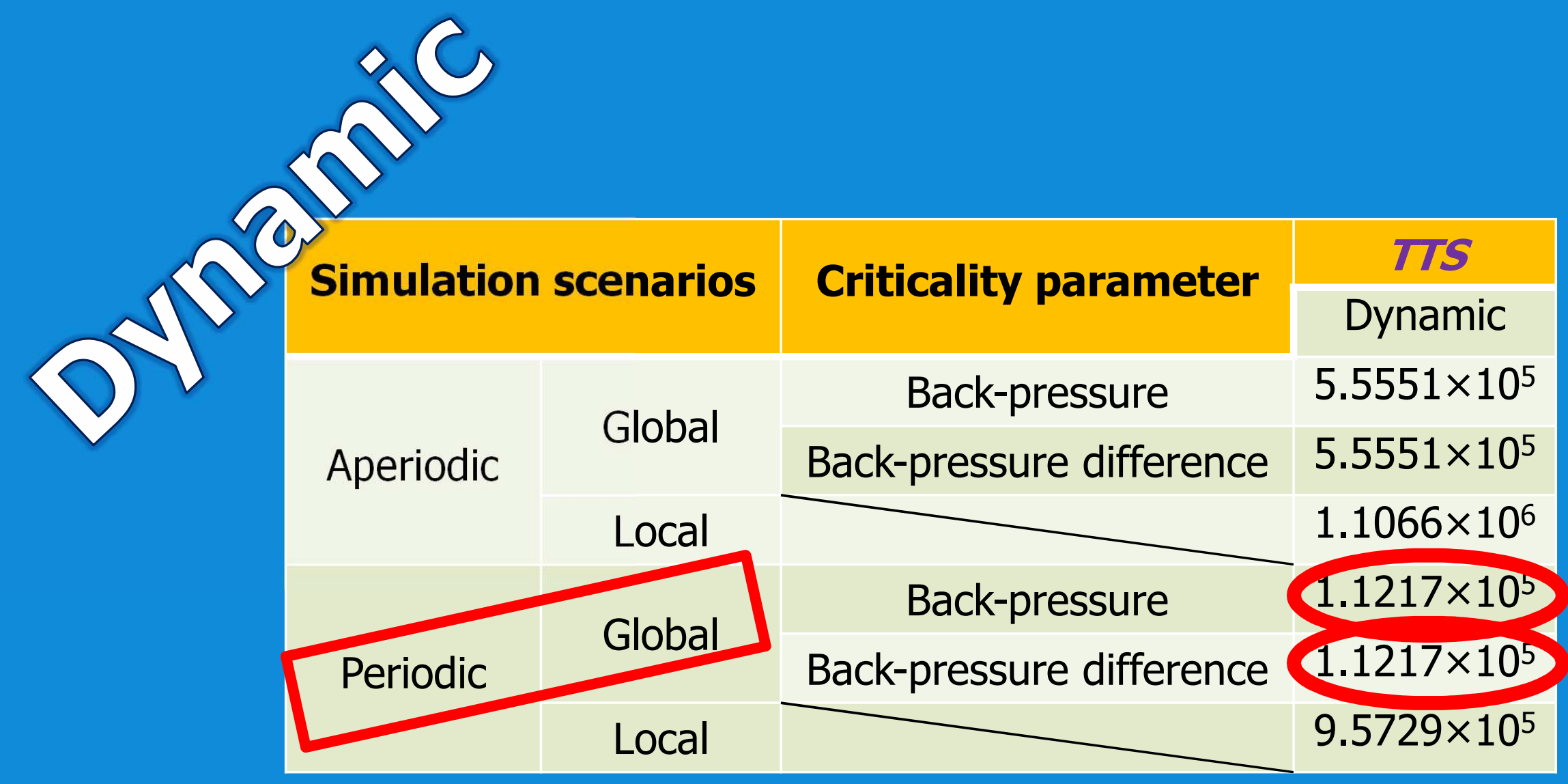




\section{Conclusion}

We conclude a slot time calculation approach to extend the basic backpressure signal control strategy. This approach takes the all red time into consideration and overcomes the low robustness of the basic one. 


\section{Thank you !}

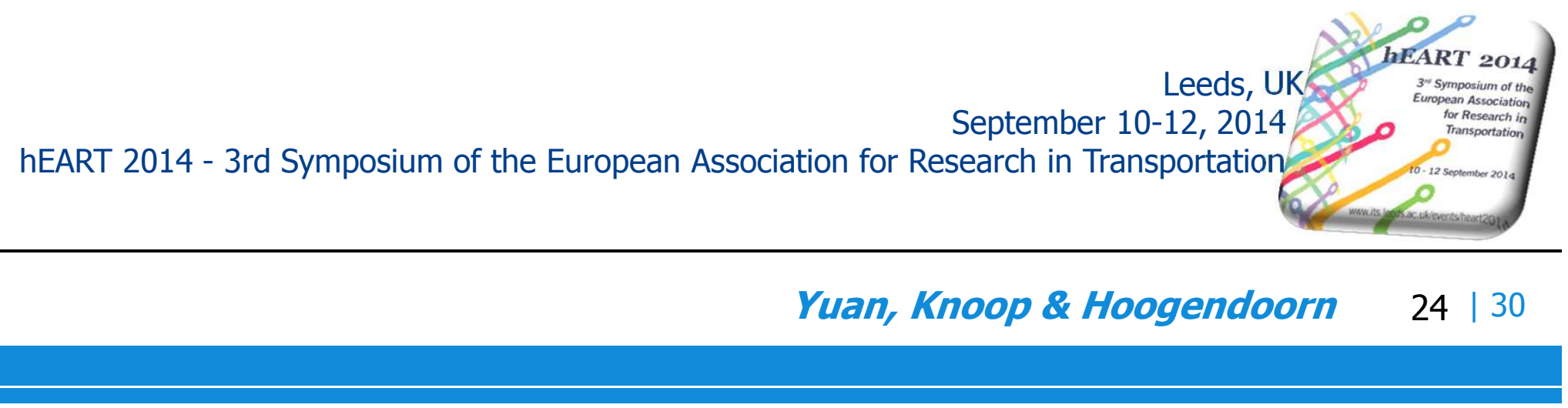

